\title{
Clinical features of myasthenia gravis in elderly and senile patients
}

\author{
Larisa Volkova $^{1}$, Marina Toporkova ${ }^{2}$, Alla Galunova ${ }^{3}$, and Lidia Sokolova ${ }^{1}$ \\ ${ }^{1}$ Department of nervous diseases, neurosurgery and medical genetics, Ural State Medical University, \\ 620028, Repina Str., 3, Ekaterinburg, Russia \\ 2 Neurology department, Medical association "New Hospital", 620109, Zavodskaya Str., 29, \\ Ekaterinburg, Russia \\ ${ }^{3}$ Neurology department ,GAUZ SO “SOKB N 1", 620120, Volgogradskaya Str., 185, Ekaterinburg, \\ Russia
}

\begin{abstract}
The prevalence of myasthenia gravis is $10-24$ cases per 100,000 population. The debut of the disease is possible at any age: from infancy to old age. In the literature, the definition of "myasthenia gravis with late onset" - at the age of 60 years and older, which is $20-46 \%$ of all cases. In this regard, the disease is often combined with a burdened somatic history, which makes it difficult to timely diagnosis and treatment. This group of elderly patients accounts for the majority of diagnostic errors in the diagnosis of myasthenia gravis. This study is devoted to the study of the features of the onset and course of myasthenia gravis in elderly and senile people.
\end{abstract}

\section{Introduction}

Myasthenia gravis is a chronic autoimmune neuromuscular disease [1]. The disease is clinically characterized by weakness and pathological muscle fatigue due to the formation of autoantibodies to various antigenic structures of the neuromuscular synapse $[2,3]$.

The prevalence of myasthenia gravis is $10-24$ cases per 100,000 population. According to statistics, in $75 \%$ of cases, the disease debuts at the age of 15 to 30 years [1-3], but in the literature of recent years, the definition of "myasthenia gravis with a late onset" - at the age of 60 years and older, which is $20-46 \%$ of all cases [4]. The ratio of men and women is $1: 3$, and in old age, men get sick as often as women [5]. The course of the disease at a young age can often be aggravated by a combination with other autoimmune disorders: pathology of the thyroid gland (hyper- or hypothyroidism), rheumatoid arthritis or diabetes mellitus [6,7]. Myasthenia gravis with late onset is characterized by a combination with chronic somatic pathology, which makes timely diagnosis and treatment difficult [8-10]. The group of elderly patients accounts for the majority of diagnostic errors in the diagnosis of myasthenia gravis. 


\section{Materials and Methods}

Over a two year period (2018-2019) a retrospective analysis was conducted of 24 cases of medical histories of patients with generalized myasthenia gravis in the decompensation stage (18 women and 6 men) aged over 60 years who were treated in the neurological department of MA «New hospital» and GAUZ SO "SOKB\#1" was conducted. The anamnesis of the disease, neurological and somatic status were studied in detail and systematized. The severity of the disease was assessed according to the MGFA clinical classification. The results of computed tomography (CT) of the chest and anterior mediastinal organs were analyzed in all patients. The results of electroneuromyography (ENMG) were analyzed, and serological tests for the determination of antibodies to acetylcholine receptors were performed in all patients.

\section{Results and Discussion}

Among the examined patients with late onset of myasthenia gravis, the majority were aged $60-75$ years -18 people $(75 \%), 25 \%$ - senile age $-75-90$ years. The average age of patients is 73 years.

The duration of the disease at the time of the study was about a year in 5 patients (20.8\%), 5-10 years - in 17 patients $(70.8 \%)$ and in 2 patients $(8.4 \%)$ more than 10 years.

In the study group, all patients were diagnosed with myasthenia gravis after an average of 1-6 months (in some patients after a year) from the beginning of clinical manifestations, which is associated with an incorrect interpretation of weakness and fatigue in less active and mobile elderly people 15 patients $(62.5 \%)$ or an erroneous diagnosis of acute st in 7 patients $(29.2 \%)$, more often in the vertebro-basilar system due to the development of oculomotor disorders.

In the future, the diagnosis of myasthenia gravis was based on at least three criteria out of four: clinical, pharmacological, electroneuromyographic, and/or immunological. All patients were given a pharmacological test with neostigmine methylsulfate in a dose of $2 \mathrm{ml}$ $0.05 \%$ solution subcutaneously. The majority patients $(20-83.3 \%)$ tested positive with full (12-50\%), incomplete $(6-25 \%)$ or partial $(2-8.3 \%)$ compensation for motor disorders, but 4 patients $(16.7 \%)$ had a pharmacological test that was considered doubtful.

In the majority of the patients, myasthenia gravis debuted in a local form in 18 ( $75 \%$ ) patients (14 with ocular symptoms, 4 with weakness of bulbar muscle groups), generalized muscle weakness (mainly in skeletal muscles) was the first symptom of the disease in 6 $(25 \%)$ patients. Later, all patients with local manifestations of the disease developed a generalized form of myasthenia gravis, more often during the first 2-3 years of the disease 16 patients, only 2 patients 10 years after the onset of symptoms after a period of long remission.

According to the results of ENMG using the method of indirect rhythmic muscle stimulation with registration of the M-response, a positive decrement test (17-39\%) was detected in all 24 patients, pathognomonic for the diagnosis of myasthenia gravis.

CT scans of the chest and anterior mediastinum revealed no thymus pathology in the majority of 20 patients $(83.3 \%)$. Thymoma was observed in 4 patients $(16.7 \%)$, in this regard, 2 patients later underwent surgical treatment (thymectomy) and 2 patients radiation therapy - in all cases with improved condition, partial recovery of motor function and performance with the support of moderate doses of pyridostigmine bromide and glucocorticosteroids (GCS).

In almost all patients $-21(87.5 \%)$ - the course of myasthenia gravis was burdened with concomitant pathology. Arterial hypertension was most frequently registered - in 19 patients $(79.2 \%), 10$ patients $(41.7 \%)$ were diagnosed with coronary heart disease, type 2 
diabetes and hypothyroidism were detected with the same frequency - in 9 patients (37.5\%), in rare cases there was a history of chronic obstructive pulmonary disease, gastric ulcer and malignant neoplasm (breast cancer). The most severe course of myasthenia gravis was observed in 8 patients who had 3 comorbidities diseases (hypertension, hypothyroidism and diabetes mellitus).

According to the results of serological blood analysis for the determination of antibodies to acetylcholine receptors, two groups were identified: seropositive with an antibody concentration of $1.5-29.3 \mathrm{nmol} / \mathrm{l}$ - 19 people $(79.2 \%)$, seronegative -5 patients $(20.8 \%)$. However, there was no correlation between the presence or level of antibodies and the severity of the disease.

All patients daily receive anticholinesterase drugs - pyridostigmine bromide $60 \mathrm{mg}$ 2-3 times a day, glucocorticosteroids - methylprednisolone in an individual dose -18 patients $(75 \%)$, in 6 patients due to the severity of the disease - cytostatics - azathioprine $50-100 \mathrm{mg}$ $(25 \%)$ were used in therapy. During the course of the disease, plasmapheresis was performed in 10 patients $(41.7 \%)$ due to impaired breathing and swallowing, progressive muscle weakness as a result of decompensation of severe myasthenia gravis or against the background of a developed myasthenic crisis. In most patients, decompensation of the disease was due to infectious disease (SARS, intestinal infection) - 10 patients $(41,7 \%)$, stress in 6 patients $(25 \%)$ and physical activity in 3 patients $(12,5 \%), 5$ cases $(20,8 \%)$ the reason for decompensation was the cancellation or drastic dose reduction of basic therapy by the patient without consulting with doctor on a background of full recovery of symptoms is more often observed at the onset of the disease with local symptoms of eye disease.

When evaluating the clinical picture at the time of admission according to the MGFA classification, mild severity of class $2 \mathrm{~A}$ was observed in 7 (29.2\%) patients. Moderate myasthenic disorders of class 3A were observed in 7 patients $(29.2 \%)$. In $10(41.7 \%)$ patients, a severe course of myasthenia gravis (myasthenic crisis, decompensation) of class 5 was detected with impaired respiratory and swallowing function, requiring respiratory support (ventilator) and nutrition through a nasogastric probe with simultaneous repeated plasmapheresis sessions. During treatment in hospital in 6 patients $(25 \%)$, the severe course of the disease, often with a ventilator, was complicated by the formation of pneumonia (aspiration or ventilation-associated).

\section{Conclusions}

1. Myasthenia gravis with "late onset" in most cases (75\%) develops in the elderly age of 60-75 years, often against the background of severe somatic pathology. Combined pathology (arterial hypertension, diabetes mellitus, hypothyroidism) significantly worsens the course of the disease, contributing to the development of decompensation against the background of respiratory infection.

2. It should be noted frequent diagnostic errors in the diagnosis of myasthenia gravis in patients of elderly and senile age. During 6-12 months symptoms explained by doctors decompensated somatic pathology. A third of patients were mistakenly diagnosed with acute stroke, most often in the vertebro-basilar system due to the development of oculomotor disorders.

3. Despite the fact that in $75 \%$ of elderly and senile patients, the first symptoms of the disease were manifestations of local muscle weakness of the ocular and(or) bulbar muscle groups, all patients later showed progression of the disease with generalization of muscle weakness.

4. $20.8 \%$ of patients with myasthenia gravis in in the elderly had seronegative tests for antibodies to acetylcholine receptors. There were probably patients in this group with other autoantibodies to muscle-specific tyrosine kinase (MuSK-Ab) or ryanodine receptors 
(RyR), but unfortunately, diagnostics for the level of other antibodies is not currently available in clinical practice.

5. In a quarter of patients, decompensation of the disease or myasthenic crisis required resuscitation AIDS and plasmapheresis. Most often, the reason was an independent cancellation or sharp reduction in doses of basic therapy of the disease. In this regard, you should pay attention to neurologists who monitor the management of patients at the outpatient stage, the prevention of infections and the patient's psychological mood for longterm treatment.

\section{References}

1. A.G. Sanadze, Myasthenia and myasthenic syndromes: a guide for doctors, 256 (M.: Littera, 2012)

2. V.V. Ponomarev, Neurological journal, 1, 19-23 (2010)

3. T.M. Alekseeva, V.D. Kosachev, A.N. Khalmurzina, Neuromuscular diseases Jour., 6(3), 10-16 (2016)

4. E.M. Hernandez Ocampo, J.M. Aragones Pascual, J. Am. Geriatr. Soc., 60(12), 2379 (2012)

5. J. Sussman et al., Pract. Neurol., 15, 199-206 (2015)

6. Z. Pakzad, T. Aziz, J. Oger, Neurology, 76(17), 1526-8 (2011)

7. J.A. Aarli. N.Y. Ann, Acad Sci., 1132, 238-43 (2008)

8. A. Evoli, A.P. Batocchi, C. Minisci et al., J. Am. Geriatr. Soc., 48(11), 1442-8 (2000)

9. N. Basic-Jukic, B. Brunetta, P. Kes, Ther. Apher. Dial., 14(2), 161-5 (2010)

10. F. Romi, N.E. Gilhus, J.E. Varhaug et al., Eur. J. Neurol., 9(1), 55-61(2002) 